\section{Intestinal graft-versus-host-disease staging by video capsule endoscopy}

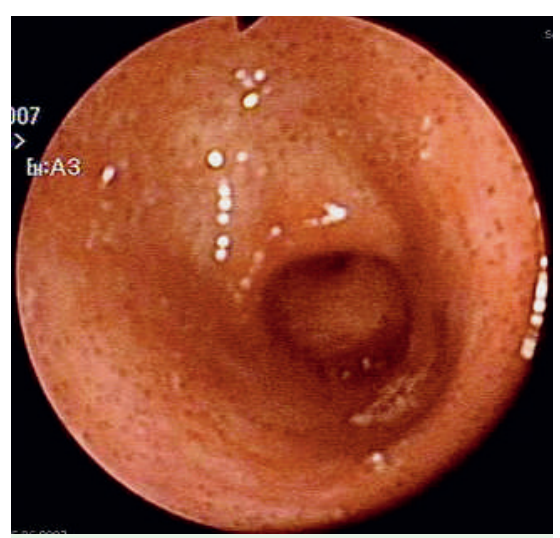

Fig. 1 Endoscopic appearance of severe diffuse hemorrhagic inflammation of the sigmoid colon.

Graft-versus-host disease (GvHD) is a leading cause of morbidity and mortality in patients who have undergone allogeneic bone marrow or peripheral blood stem cell transplantation (BMT/PBSCT) [1]. About $20-50 \%$ of patients who have received BMT/PBSCT develop symptoms of acute GvHD [2]. GvHD most often affects the skin, liver, and gastrointestinal tract. In severe intestinal GvHD broad ulcerative lesions develop, leading to diarrhea, malabsorption, intestinal hemorrhage, and sepsis [3]. The gold standard in the diagnosis of intestinal GvHD is upper and lower gastrointestinal endoscopy with histological validation [4].

A 57-year-old woman with acute myeloid leukemia who had recently received a PBSCT from an HLA-identical sibling suffered severe hemorrhagic diarrhea. The symptoms started 12 days after the transplant. The diagnosis of GvHD was histologically and endoscopically confirmed by sigmoidoscopy (CF-H180AI/L; Olympus Co. Ltd., Tokyo, Japan) ( Fig. 1). Esophagogastroduodenoscopy did not reveal relevant pathologic findings.

Since the patient's condition rapidly deteriorated, a total colectomy was discussed as a last therapeutic option following the failure of several immunosuppressive drug regimens including corticosteroids, cyclosporine, mycophenolate mofetil, pentostatin, infliximab, and antithymocyte globulin. Video capsule endoscopy (PillCam SB; Given Imaging Ltd., Yoq-

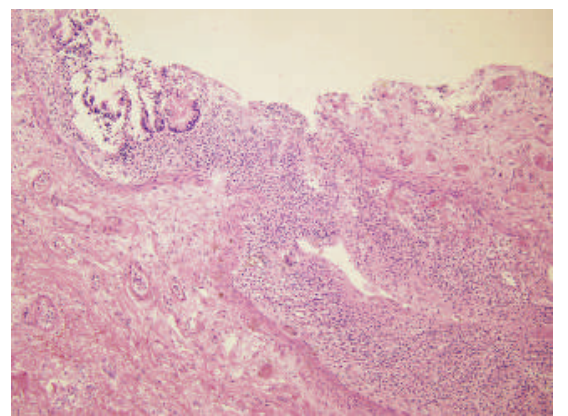

Fig. 2 Histological section (H\&E staining) showing autolytic mucosa of the small bowel with acute fibrinous and chronic inflammation. Pronounced submucosal edema and dilated capillary vessels due to graft-versus-host disease.

neam, Israel) carried out to evaluate small-bowel involvement in the GvHD revealed continuous severe hemorrhagic inflammation of the entire small intestine starting from the proximal jejunum ( $\bullet$ Video 1) and ending in the terminal ileum ( $\bullet$ Video 2 ).

Video capsule endoscopy proved to be a successful minimally invasive diagnostic method, accurately visualizing the involvement of the small intestine in severe GvHD, obviating the necessity for diagnostic surgical exploration in a clinically unstable patient.

The patient died of multiorgan failure due to GvHD. Histological analysis of the intestine confirmed severe GvHD involving the entire small intestine and colon (๑ Fig. 2). We conclude that video capsule endoscopy is suitable for staging intestinal GvHD especially in those patients unable to tolerate invasive diagnostic measures such as double-balloon enteroscopy or surgery.

\section{Video 1}

Sequence of video capsule endoscopy of the proximal jejunum showing severe hemorrhagic mucosal inflammation and broad ablation of the mucosal surface.

\section{Video 2}

Sequence of video capsule endoscopy of the ileum with large amounts of intraluminal blood due to severe graft-versus-host disease.
T. Meister ${ }^{1}$, H. Heinzow ${ }^{1}$, G. Bisping ${ }^{2}$, M. Stelljes ${ }^{2}$, B. Schulte ${ }^{3}$, W. E. Berdel ${ }^{2}$, J. Kienast ${ }^{2}$, W. Domschke ${ }^{1}$, H. Ullerich ${ }^{1}$ Department of Medicine B, University of Münster, Münster, Germany

2 Department of Medicine A, University of Münster, Münster, Germany

Gerhard-Domagk-Institut of Pathology, University of Münster, Münster,

Germany

\section{References}

1 Vogelsang GB, Lee L, Bensen-Kennedy DM. Pathogenesis and treatment of graft-versus-host disease after bone marrow transplant. Annu Rev Med 2003; 54: 29-52

2 Remberger $M$, Persson $U$, Hauzenberger D et al. An association between human leucocyte antigen alleles and acute and chronic graft-versus-host disease after allogeneic haematopoietic stem cell transplantation. Br J Haematol 2002; 119: 751 - 759

3 Martin PJ, McDonald GB, Sanders JE et al. Increasingly frequent diagnosis of acute gastrointestinal graft-versus-host disease after allogeneic hematopoietic cell transplantation. Biol Blood Marrow Transplant 2004; 10: $320-327$

4 Cruz-Correa M, Poonawala A, Abraham SC et al. Endoscopic findings predict the histologic diagnosis in gastrointestinal graft-versus-host disease. Endoscopy 2002; 34: $808-813$

Bibliography

DOI $10.1055 / \mathrm{s}-2007-995767$

Endoscopy 2008; 40: E144

(c) Georg Thieme Verlag KG Stuttgart · New York . ISSN 0013-726X

Corresponding author

H. Ullerich, MD

Department of Medicine B

University of Münster

Albert-Schweitzer-Str. 33

48149 Münster

Germany

Fax: +49-251-8347576

ullerih@mednet.uni-muenster.de 